\title{
Resonance effects in the Raman scattering of monolayer and few-layer $\mathrm{MoSe}_{2}$
}

\author{
P. Soubelet, ${ }^{1}$ A. E. Bruchhausen, ${ }^{1}$ A. Fainstein, ${ }^{1}$ K. Nogajewski, ${ }^{2}$ and C. Faugeras ${ }^{2, *}$ \\ ${ }^{1}$ Instituto Balseiro and Centro Atómico Bariloche, C.N.E.A., R8402AGP Bariloche, Río Negro, Argentina \\ ${ }^{2}$ LNCMI (CNRS, UJF, UPS, INSA), BP 166, 38042 Grenoble Cedex 9, France
}

(Received 5 November 2015; published 5 April 2016)

\begin{abstract}
Using resonant Raman scattering spectroscopy with 25 different laser lines, we describe the Raman scattering spectra of monolayer and multilayer $2 \mathrm{H}$-molybdenum diselenide $\left(\mathrm{MoSe}_{2}\right)$ as well as the different resonances affecting the most pronounced features. For high-energy phonons, both $A$ - and $E$-symmetry type phonons present resonances with $A$ and $B$ excitons of $\mathrm{MoSe}_{2}$ together with a marked increase of intensity when exciting at higher energy, close to the $C$-exciton energy. We observe symmetry-dependent exciton-phonon coupling affecting mainly the low-energy rigid layer phonon modes. The shear mode for multilayer displays a pronounced resonance with the $C$ exciton while the breathing mode has an intensity that grows with the excitation laser energy, indicating a resonance with electronic excitations at energies higher than that of the $C$ exciton.
\end{abstract}

DOI: 10.1103/PhysRevB.93.155407

Layered transition-metal dichalcogenides $\left(M X_{2}\right)$ are today the subject of intense studies because of the possibility to isolate a single monolayer, a two-dimensional (2D) crystal, out of the bulk material [1,2]. This family of materials includes semiconducting compounds $\left(M X_{2}\right.$ with $M=$ Mo or $\mathrm{W}$ and $X=\mathrm{S}, \mathrm{Se}$, or $\mathrm{Te})$. They offer an interesting platform to explore purely two-dimensional excitons, phonons, and to study the interplay between the spin and valley physics [3,4]. Monolayers of these materials can be transferred on adapted substrates for optical or transport investigations. In contrast to gapless graphene, their band gap of 1-2 eV allows for the construction of transistors [5] and of photodetectors [6]. Even though the room-temperature electronic mobilities they exhibit reach only the level of few hundreds $\mathrm{cm}^{2} \mathrm{~V}^{-1} \mathrm{~s}^{-1}$, orders of magnitude below what is achieved in conventional III-V or II-VI semiconductor quantum wells, their flexibility and transparency are extremely appealing for future electronic and optoelectronic applications. For instance, they are used as building blocks of van der Waals heterostructures [7-9], which cannot be controlled without a deep knowledge of the phonon modes and of the electron-phonon interaction in the individual layers. It is also worth to point out that the photoluminescence (PL) of $M X_{2}$ monolayers arises from tightly bound excitons $[10,11]$, with binding energies close to $400 \mathrm{meV}$, and hence comparable to those usually observed in molecules or in carbon nanotubes [12,13].

Raman scattering plays an important role in the rapid development of the field of research of $2 \mathrm{D}$ crystals as it provides, for most layered materials, a fast and noninvasive tool to determine the number of layers of a given specimen [14]: When the thickness of a $M X_{2}$ flake is reduced, the energy of some optical phonons changes in a way that can be traced using standard techniques. Resonant Raman scattering is a powerful technique to explore electronic properties of materials. The resonant enhancement of Raman scattering signals when the laser energy is tuned to particular values corresponding to electronic excitations of the investigated system is a well-established tool to perform, for instance, the spectroscopy of weakly radiative excitations. This technique

*clement.faugeras@1ncmi.cnrs.fr has been applied to $\mathrm{MoS}_{2}$ thin layers, probably the most explored layered $M X_{2}$ so far, and to $\mathrm{WSe}_{2}$ [15-19].

The Raman spectrum of bulk $2 \mathrm{H}-\mathrm{MoSe}_{2}$ has been measured in 1980 [20], allowing for the identification of the main Ramanactive phonon modes of this compound. Since that time, only few studies reported the Raman spectrum of thin layers of this material [21-23]. Although they provided the description of the main phonon modes, a complete characterization of the Raman scattering spectrum of thin layers of $\mathrm{MoSe}_{2}$ is still lacking. The phonon band structure of monolayer $\mathrm{MoSe}_{2}$ has recently been calculated by $a b$ initio techniques [24], and this compound appears to be distinct from other members of the $M X_{2}$ family. All these materials present six optical and three acoustical phonon branches, but, in contrast to the case of $\mathrm{MoS}_{2}, \mathrm{WS}_{2}$, or $\mathrm{WSe}_{2}$ [25], according to these calculations, in $\mathrm{MoSe}_{2}$ the $A_{1}^{\prime}$ mode is located between the $E^{\prime}$ and $E^{\prime \prime}$ modes and is not the highest Raman-active mode at the $\Gamma$ point of the phonon band structure. The phonon mode of $E$ symmetry around $290 \mathrm{~cm}^{-1}$, measured at $632.8 \mathrm{~nm}$, has a much weaker intensity than the $A_{1}$ mode [23] close to $243 \mathrm{~cm}^{-1}$. The $E^{\prime}$ mode is split over the whole phonon Brillouin zone, with a splitting of $\sim 4 \mathrm{~cm}^{-1}$ at the $\Gamma$ point. These two $E^{\prime}$ phonons are of longitudinal (LO) and of transverse character (TO), and their splitting comes from the polar character of $\mathrm{MoSe}_{2}$.

Resonant studies with a large number of laser lines spanning from the near infrared to the ultraviolet are seldom done, and this is particularly difficult and absent in the literature when low-energy excitations are involved. It is such a systematic and comprehensive study that is being reported here, for a model $M X_{2}$ material $\left(\mathrm{MoSe}_{2}\right)$ from monolayer to multilayer structures. We present a resonant Raman scattering investigation of monolayer and multilayer $2 \mathrm{H}-\mathrm{MoSe}_{2}$ using 25 different laser excitation lines from $458 \mathrm{~nm}(2.7 \mathrm{eV})$ to $825 \mathrm{~nm}(1.5 \mathrm{eV})$, which span the energies of the three different excitons $A, B$, and $C$, located close to $1.54,1.74$, and $2.61 \mathrm{eV}$, respectively [26], and including both high- and low-energy vibrations. In the first part, we focus on monolayer $\mathrm{MoSe}_{2}$ (1L-MoSe $e_{2}$, discussing in particular the identification of the main Raman scattering features and their excitation profiles. We then address the case of multilayer $\mathrm{MoSe}_{2}\left(N \mathrm{~L}-\mathrm{MoSe}_{2}\right.$ where $N$ is the number of layers) and describe the phonon modes which are only Raman active in multilayers. These 
modes have been observed in $\mathrm{MoTe}_{2}$ [27-29], $\mathrm{MoS}_{2}$ [18], $\mathrm{WSe}_{2}[30,31]$, and we propose here their proper identification in $\mathrm{MoSe}_{2}$, based on polarization-resolved measurement and on resonant Raman scattering experiments. We present the excitation profiles of the main Raman features, including the low-energy shear and breathing modes in the case of $2 \mathrm{~L}-\mathrm{MoSe}_{2}$, and provide evidence, for some of these modes, of an increase of the scattering efficiency at energies beyond that of the $C$ exciton.

Raman scattering experiments were performed at room temperature in the backscattering geometry with a triplegrating spectrometer equipped with a nitrogen-cooled charge coupled device (CCD) camera. Laser excitation was provided by a mixed $\mathrm{Ar} / \mathrm{Kr}$ laser (from 458 to $676 \mathrm{~nm}$ ) and by a Ti:sapphire laser (from 680 to $825 \mathrm{~nm}$ ). Laser lines were filtered with a grating used in the Littrow configuration. A $50 \times$ objective was used to focus the excitation laser down to a spot of $2 \mu \mathrm{m}$, with optical power of $0.4 \mathrm{~mW}$. Thin flakes of $\mathrm{MoSe}_{2}$ were first exfoliated from bulk MoSe $e_{2}$ purchased from $H Q$ graphene and then deposited on a $\mathrm{Si} / \mathrm{SiO}_{2}$ substrate with a $\mathrm{SiO}_{2}$ thickness of $90 \mathrm{~nm}$. Flakes composed of 1 to 8 layers were identified by their different optical contrast contrast (calibrated with AFM measurements on other flakes) and then by low-energy Raman scattering and photoluminescence experiments. Raman scattering spectra were normalized by the integrated intensity of the silicon peak and by its resonant excitation wavelength dependence [32], and by the effect of interferences in the $\mathrm{SiO}_{2}$ layer [33] (see Appendix). The luminescence background in the case of $1 \mathrm{~L}$ and $2 \mathrm{~L}$ has been removed from the presented spectra. In the following, the intensity of each mode is normalized to its maximum value observed in the investigated excitation energy range. We are interested in the independent evolution of the different modes and not by their relative intensities. The vibration patterns of $M X_{2}$ have been described in many recent reports [34,35]. Bulk $2 \mathrm{H}-M X_{2}$ belongs to the $D_{6 h}$ point group, while their odd and even few-layer counterparts represent the $D_{3 h}$ and $D_{3 d}$ point groups, respectively. This implies a change of the symmetry of the phonon modes depending on the number of layers. In what follows, we will use the previously introduced notation [18], indicating the symmetry of the different phonon modes by a double notation corresponding to the odd and even number of layers.

In Figs. 1(a)-1(c), we present three Raman scattering spectra of monolayer $\mathrm{MoSe}_{2}$ measured at $465 \mathrm{~nm}$ [Fig. 1(a)], at $676 \mathrm{~nm}$ [Fig. 1(b)], and at $735 \mathrm{~nm}$ [Fig. 1(c)]. The scale of these spectra is set in order to compare the position of the different features and normalized spectra are presented in Fig. 1(d). With a 465-nm excitation, the most prominent feature is the $A_{1}^{\prime}$ mode observed at $242 \mathrm{~cm}^{-1}$ in $1 \mathrm{~L}-\mathrm{MoSe}_{2}$. Its intensity is 10 times bigger than the intensity of all other Raman scattering features. The $E^{\prime}$ mode, predicted to be Raman active in Ref. [24], is also observed at $288 \mathrm{~cm}^{-1}$. These are the two main features of the Raman scattering spectrum of $1 \mathrm{~L}-\mathrm{MoSe}_{2}$ $[21-23,36]$.

A more detailed investigation shows that, similar to other $M X_{2}$ [15,37-40] compounds, acoustical phonons also contribute to the Raman scattering spectrum of $1 \mathrm{~L}-\mathrm{MoSe}_{2}$ and that depending on the excitation wavelength, such contribution can be strongly enhanced (see, e.g., the spectra measured using
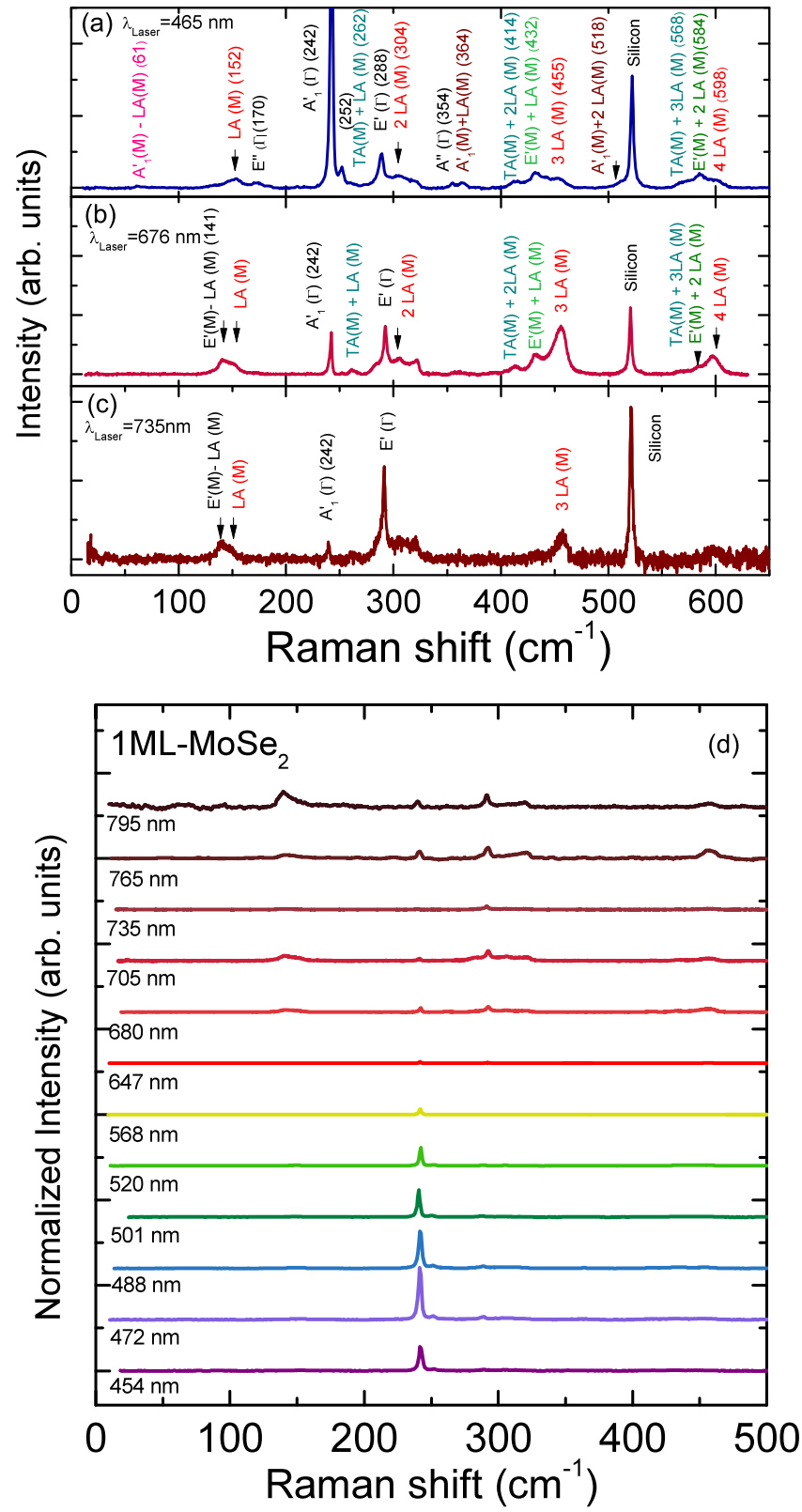

FIG. 1. Raman scattering spectra of monolayer $\mathrm{MoSe}_{2}$ measured with (a) 465-, (b) 676-, and (c) 735-nm laser excitation. The main Raman scattering features are identified in the figure and the intensity scale is set in order to see the features for all excitations wavelengths. (d) Normalized Raman scattering spectra for the monolayer.

676- and 735-nm laser excitations). Because the dispersion of acoustical phonons close to the $M$ and $K$ points is rather flat [24], the associated density of states is peaked close to this energy and defect-mediated phonon scattering is favored. Most of these modes are observed for the first time and, following the large body of Raman scattering studies of such multi-phonon features in other $M X_{2}$ compounds and theoretical calculations of the phonon band structure, we propose the following assignment. We observe the longitudinal acoustical phonon from the $M-K$ points $[\mathrm{LA}(M)]$ at $152 \mathrm{~cm}^{-1}$ and its first overtones $[2 \mathrm{LA}(M), 3 \mathrm{LA}(M)$, and $4 \mathrm{LA}(M)]$. Next, we see a combination of optical and acoustical phonons from the $M$ point, 


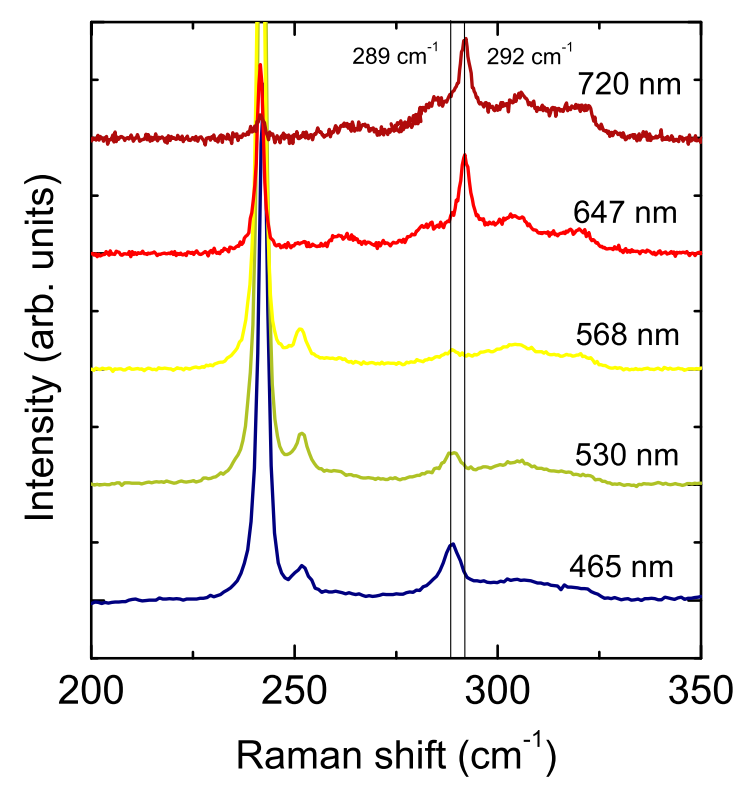

FIG. 2. Raman scattering spectra of monolayer $\mathrm{MoSe}_{2}$ measured with 465-, 530, 568-, 647-, and 720-nm laser excitation showing the selective excitation of the two $E^{\prime}$ optical phonons.

namely, $A_{1}^{\prime}(\mathrm{M}) \pm \mathrm{LA}(M)$ at 61 and $364 \mathrm{~cm}^{-1}$, respectively, $A_{1}^{\prime}(M)+2 \mathrm{LA}(M)$ at $518 \mathrm{~cm}^{-1}$, and $E^{\prime}(M)+\mathrm{LA}(M)$ as well as $E^{\prime}(M)+2 \mathrm{LA}(M)$ at 432 and $584 \mathrm{~cm}^{-1}$, respectively. One can also notice in our experimental data the TA $(M)+X \operatorname{LA}(M)$ with $X=1$ to 3 at 262,414 , and $568 \mathrm{~cm}^{-1}$. These acoustical phonon features are observed on freshly exfoliated $\mathrm{MoSe}_{2}$ flakes, and also, with similar intensity, on flakes produced six months before.

An additional Raman scattering feature appears at $252 \mathrm{~cm}^{-1}$ on all samples, monolayers, or multilayers. Polarizationresolved measurements show that this feature is of the $A$-type symmetry, and it follows the same resonances, discussed in the following, as the main $A_{1}^{\prime}$ feature at $242 \mathrm{~cm}^{-1}$. At high excitation energy, we also observe some traces of the modes $E^{\prime \prime}(\Gamma)$ and $A^{\prime \prime}(\Gamma)$ at 170 and $354 \mathrm{~cm}^{-1}$, respectively $[24,25]$, which are normally forbidden for monolayers in backscattering geometry.

When decreasing the excitation energy using 676- or 735-nm excitation [Figs. 1(b) and 1(c)], the Raman scattering spectrum of $1 \mathrm{~L}-\mathrm{MoSe}_{2}$ changes drastically: the $A_{1}^{\prime}$ feature at $242 \mathrm{~cm}^{-1}$, which is prominent at higher excitation energy, now has an intensity comparable to that of the acoustical phonon features and of their overtones. The mode $\mathrm{TA}(M)+\mathrm{LA}(M)$, at $262 \mathrm{~cm}^{-1}$, and the $\mathrm{LA}(M)$ overtones also become particularly well visible in this excitation energy regime. The $E^{\prime}(\Gamma)$ phonon, previously observed at $289 \mathrm{~cm}^{-1}$, now appears at slightly higher energy $292 \mathrm{~cm}^{-1}$, which we tentatively interpret, based on the phonon band structure calculations [24], as the selective excitation, for different excitation laser energies, of the lower-energy TO mode or of the upper-energy LO mode [24]. This crossover is presented in more details in Fig. 2. The lower component of the split $E^{\prime}$ mode, prominent for excitation wavelengths below $\sim 500 \mathrm{~nm}$, gradually disappears as the excitation wavelength increases. Above $\sim 600 \mathrm{~nm}$, the upper component of the split $E^{\prime}$ phonon appears at $292 \mathrm{~cm}^{-1}$
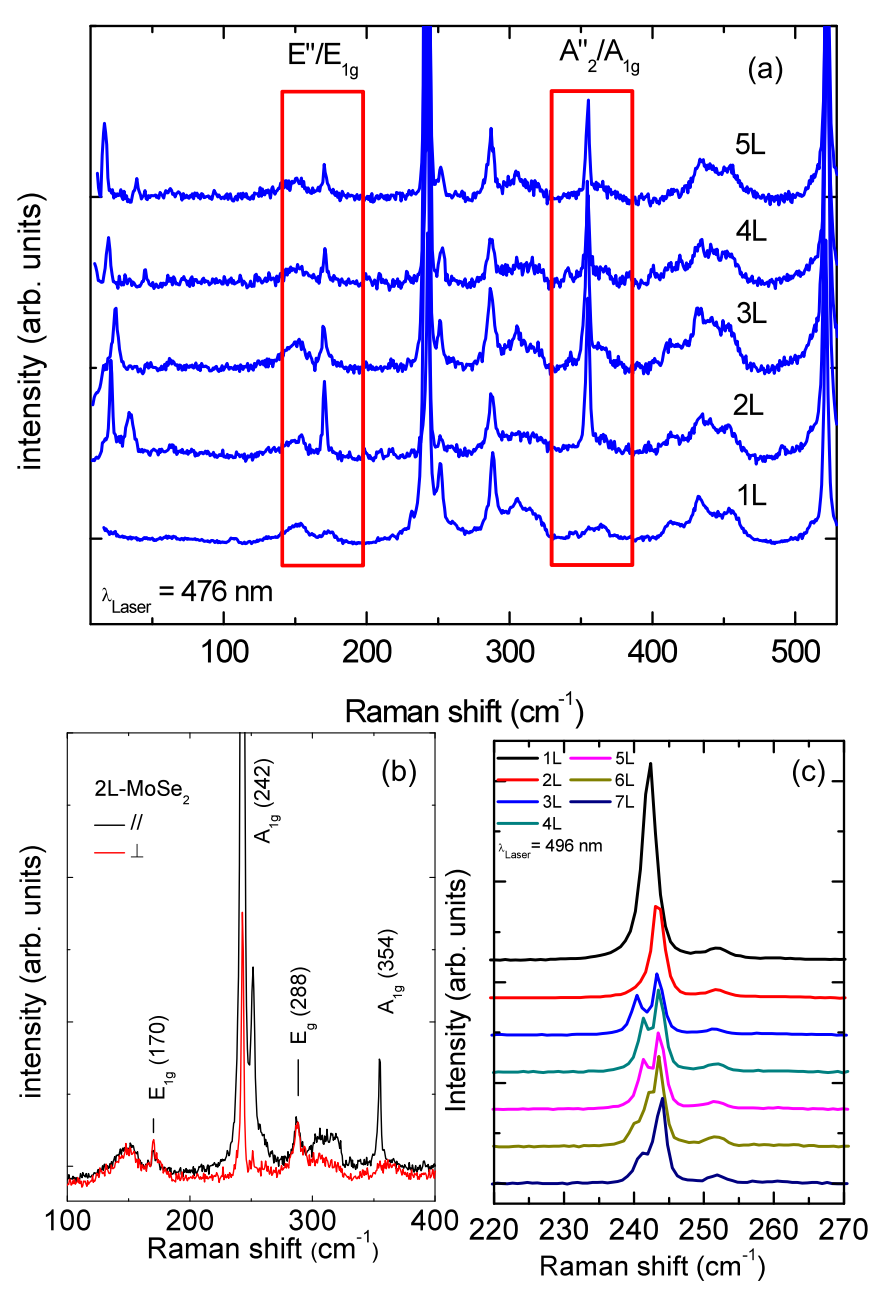

FIG. 3. (a) Raman scattering spectra of 1 to 5 layer $\mathrm{MoSe}_{2}$ recorded with the use of 465-nm excitation showing the $E^{\prime \prime}$ and $A_{2}^{\prime \prime}$ phonons at 170 and $354 \mathrm{~cm}^{-1}$, respectively. (b) Polarization-resolved Raman scattering spectra of $2 \mathrm{~L}-\mathrm{MoSe}_{2}$ measured with $514 \mathrm{~nm}$ and identifying the two $E$ - and $A$-type phonons. (c) $A_{1}^{\prime} / A_{1 g}$ feature for 1to 7-layer $\mathrm{MoSe}_{2}$ samples.

with an intensity increasing with the excitation wavelength. As will be confirmed in the following, this behavior suggests a preferential coupling of the LO phonon with the $A$ - and $B$-type excitons, while the TO phonon can only be observed when exciting at high energy, in the $C$-exciton range of energy. Such an effect, pronounced in $1 \mathrm{~L}-\mathrm{MoSe}_{2}$, is also observed in multilayers even though they do not exhibit too strong $E^{\prime} / E_{g}$ feature for low excitation energies. We do not have a microscopic explanation for this crossover and for the resonant behavior. Electronic band structure calculations, together with a phonon mode analysis, might help in understanding this effect. Such analysis is beyond the scope this experimental paper.

The Raman scattering spectrum of multilayers of $\mathrm{MoSe}_{2}$ shows some additional features. As can be seen in Fig. 3(a) for $1 \mathrm{~L}-$ to $5 \mathrm{~L}-\mathrm{MoSe}_{2}$ two additional peaks, marked by the red boxes, appear at 170 and $354 \mathrm{~cm}^{-1}$, respectively. The energy of these two modes does not depend on the number of layers. In Fig. 3(b), we present two Raman scattering spectra measured on a $2 \mathrm{~L}-\mathrm{MoSe}_{2}$ in the collinear and cross-linear 
polarization configurations. In the cross-linear configuration, the $A_{1 g}$ mode at $242 \mathrm{~cm}^{-1}$ is suppressed, together with the mode observed at $354 \mathrm{~cm}^{-1}$, showing that this mode is of $A$-type symmetry. The feature observed at $170 \mathrm{~cm}^{-1}$ is not affected by the polarization configuration and exhibits hence $E$-type symmetry. These two features observed at $170 \mathrm{~cm}^{-1}$ and at $354 \mathrm{~cm}^{-1}$ are then attributed to the zone center $E^{\prime \prime} / E_{1 g}$ and $A_{2}^{\prime \prime} / A_{1 g}$, respectively, and they are not Raman active in monolayers (even though they show up as weak features when using high excitation energies). Similar Raman scattering results have been recently obtained on $\mathrm{MoS}_{2}$ [18] and the $A_{2}^{\prime \prime} / A_{1 g}$ was observed in $\mathrm{WSe}_{2}$ [31]. These latter two modes are not observed in the $\mathrm{MoSe}_{2}$ monolayer, and, as in the case of $\mathrm{MoS}_{2}$, they only appear in multilayers for excitation energies close to the $C$-exciton energy. The effects have been clearly observed in $\mathrm{MoS}_{2}$ [18] and these authors explained this observation by the fact that, in contrast to the low-energy $A$ and $B$ excitons whose wave functions are strongly confined to individual layers, the $C$ exciton's wave function is delocalized along the whole structure $[41,42]$. As a result, the $C$ exciton can effectively couple to those phonon modes which are characteristic of multilayers and a resonance of the scattered intensity is observed for laser energies close to the $C$-exciton energy. On the other hand, features of this type do not appear at lower excitation energies, corresponding to the $A$ and $B$ excitons [18]. The observation of these two Raman features can help to quickly discriminate between monolayer and multilayer $\mathrm{MoSe}_{2}$ flakes.

A more detailed knowledge of the number of layers constituting the sample under investigation from Raman scattering experiments can be grasped from the observation of the $A_{1}^{\prime} / A_{1 g}$ feature close to $242 \mathrm{~cm}^{-1}$ [see Fig. 3(c)]. Indeed, as already reported [22], this peak has an energy that depends on the number of layers, appears in the Raman scattering spectrum as a single component feature for $1 \mathrm{~L}-\mathrm{MoSe}_{2}$ and $2 \mathrm{~L}-\mathrm{MoSe}_{2}$, splits into two components for $3 \mathrm{~L}-\mathrm{MoSe}_{2}$ and $4 \mathrm{~L}-\mathrm{MoSe}_{2}$ and into three components for $5 \mathrm{~L}-\mathrm{MoSe}_{2}$ and $6 \mathrm{~L}-\mathrm{MoSe}_{2}$. The splitting, which comes from interlayer interactions in multilayer materials $(N>2)[20,29,43]$, can be used to determine the number of layers. Close to $252 \mathrm{~cm}^{-1}$, another Raman scattering feature is observed with an energy that changes with the number of layers. Even though the proper origin of this feature is still unknown, its energy difference with the $A_{1}^{\prime} / A_{1 g}$ feature can provide an additional check for the number of layers of $\mathrm{MoSe}_{2}$ (see Appendix). In $N \mathrm{~L}-\mathrm{MoSe}_{2}$, the contribution of acoustical phonons is also observed in the Raman scattering spectrum in the form of a rich series of overtones, as already discussed in the case of the monolayer.

To complete the description of the differences between monolayers and multilayers, we note that another striking difference is seen at low vibrational frequencies, below $50 \mathrm{~cm}^{-1}$. In multilayers, as can be observed in Fig. 3(a), some additional features appear in that range with an energy that strongly depends on the number of layers. These modes are the shear and breathing modes of the multilayer structure, i.e., rigid vibrations of the whole layer with respect to each other. The evolution of these modes with increasing number of layers has recently been discussed in the case of $\mathrm{MoSe}_{2}$ [44]. One can notice from the spectra presented in Fig. 3(a) that, when measured with a 476-nm excitation, the intensity

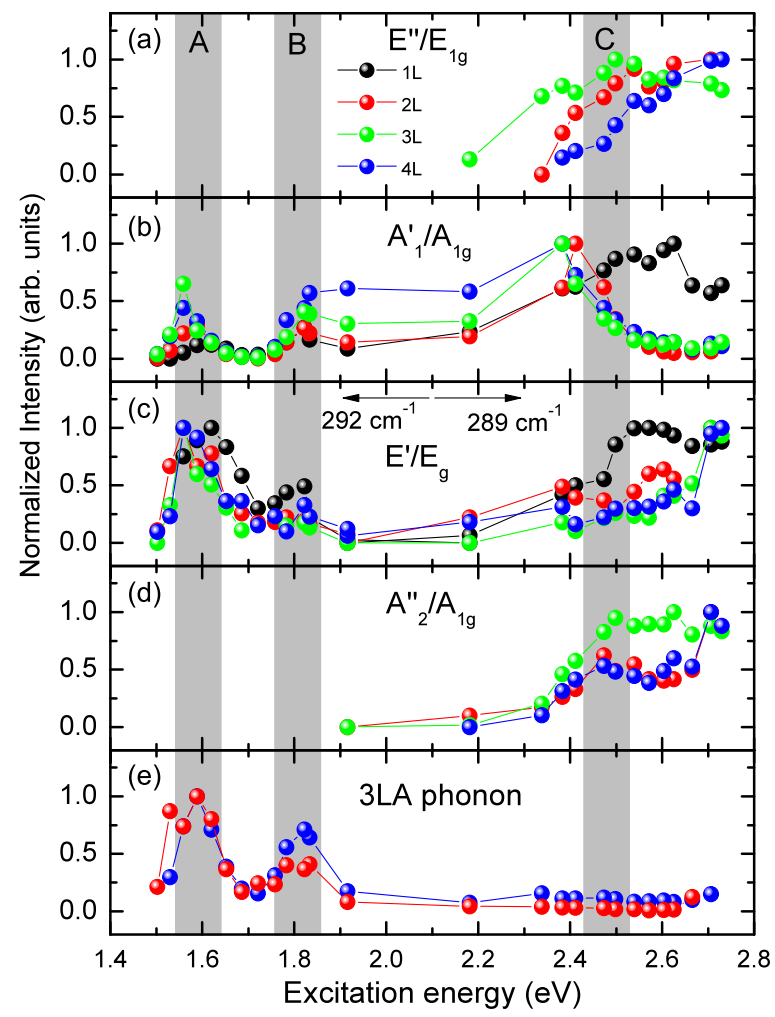

FIG. 4. Normalized intensity of (a) the $E^{\prime \prime} / E_{1 g}$ mode at $170 \mathrm{~cm}^{-1}$, (b) the $A_{1}^{\prime} / A_{1 g}$ mode at $242 \mathrm{~cm}^{-1}$, (c) the $E^{\prime} / E_{g}$ modes around $290 \mathrm{~cm}^{-1}$, (d) the $A^{\prime \prime} / A_{1 g}$ modes at $354 \mathrm{~cm}^{-1}$, and the $3 \mathrm{LA}$ phonon replica at $455 \mathrm{~cm}^{-1}$, as a function of the excitation laser energy for $1 \mathrm{~L}-\mathrm{MoSe}_{2}$ (black dots), $2 \mathrm{~L}-\mathrm{MoSe}_{2}$ (red dots), $3 \mathrm{~L}-\mathrm{MoSe}_{2}$ (green dots), and $4 \mathrm{~L}-\mathrm{MoSe}_{2}$ (blue dots). The shaded regions represent the range of energy for the $A, B$, and $C$ excitons.

of these modes is comparable with that of the $E^{\prime \prime} / E_{1 g}$ and $A_{2}^{\prime \prime} / A_{1 g}$ modes discussed previously.

When changing the excitation laser energy, all the Raman scattering features considered so far show particular resonances. These resonances for one- to four-layer flakes, are presented in Fig. 4 for the $E^{\prime \prime} / E_{1 g}$ phonon at $170 \mathrm{~cm}^{-1}$ [Fig. 4(a)], the $A_{1}^{\prime} / A_{1 g}$ phonon close to $242 \mathrm{~cm}^{-1}$ [Fig. 4(b)], the $E^{\prime} / E_{g}$ phonon close to $290 \mathrm{~cm}^{-1}$ [Fig. 4(c)], for the $A_{2}^{\prime \prime} / A_{1 g}$ phonon at $354 \mathrm{~cm}^{-1}$ [Fig. 4(d)], and for the 3LA(M) replica [Fig. 4(d)]. The $E^{\prime \prime} / E_{1 g}$ and $A_{2}^{\prime \prime} / A_{1 g}$ Raman modes at 170 and at $354 \mathrm{~cm}^{-1}$, respectively, typical for multilayers, are only observed for excitation energies above $2.2 \mathrm{eV}$. They both exhibit a resonance close to $2.5 \mathrm{eV}$. For monolayers and multilayers, we observe a resonance of the $\mathrm{LO} E^{\prime} / E_{g}$ phonon $\left(\sim 292 \mathrm{~cm}^{-1}\right)$ with the $A$ and $B$ excitons at 1.6 and $1.8 \mathrm{eV}$, respectively, while the TO $E^{\prime} / E_{g}$ phonon $\left(\sim 289 \mathrm{~cm}^{-1}\right)$ has an intensity that increases when exciting at higher energy, close to the $C$ excitons at $2.4 \mathrm{eV}$. As a result, in our experiments, these two LO and TO phonons cannot be observed simultaneously. On top of these resonances, the intensity of the TO $E^{\prime} / E_{g}$ mode appears to continuously increase when increasing the excitation laser energy. In the case of the monolayer, for which these two features are the most pronounced, the resonance appears at a slightly higher excitation energy, close to $2.6 \mathrm{eV}$. 
The main $A_{1}^{\prime} / A_{1 g}$ feature close to $242 \mathrm{~cm}^{-1}$ [Fig. 4(b)] is observed for all samples and shows for the $1 \mathrm{~L}-\mathrm{MoSe}_{2}$ a resonance at high energy close to $2.6 \mathrm{eV}$, which corresponds to the $C$-exciton energy [26]. For a higher number of layers, this high-energy resonance appears at an energy close to $2.4 \mathrm{eV}$. A second resonance is observed at $1.8 \mathrm{eV}$ and corresponds to the $B$ exciton. Within the resolution of our experiment, the energy of the resonance does not seem to depend on the number of layers. Lowering further the excitation laser energy, another resonance is observed. As for the LO $E^{\prime} / E_{g}$ feature, this resonance occurs close to $1.6 \mathrm{eV}$ and its energy strongly changes with the number of layers. The energies of the $A$ - and of the $C$-exciton energies appear to depend on the number of layers while the energy of the $B$ excitons does not. Such evolution for the $A, B$, and $C$ excitons as a function of the number of layers has recently been observed in $\mathrm{WSe}_{2}$ and in $\mathrm{MoSe}_{2}[45,46]$. These results, concerning the various resonances observed for all the phonon modes of $\mathrm{MoSe}_{2}$, appear as different as compared to those reported for $\mathrm{MoS}_{2}$, where the $A$-type phonon mode shows a resonance with the high-energy exciton only while the $E$-type modes show a resonance with exclusively the low-energy $A$ and $B$ excitons [17]. In $\mathrm{MoSe}_{2}$, the $A_{1}^{\prime} / A_{1 g}$ mode shows clear resonances with all three types of excitons, while the $E^{\prime} / E_{g}$ LO phonon displays a resonance with the $A$ and $B$ excitons and the $E^{\prime} / E_{g}$ TO phonon only appears at high excitation energy.

Some modes, namely the $E^{\prime} / E_{g}$ and the $A_{2}^{\prime \prime} / A_{1 g}$, have an intensity that, on top of the resonances observed at the exciton energies, increases strongly when increasing the excitation laser energy. This second resonance could be related to the valence band, and especially the band located $\sim 2 \mathrm{eV}$ below the highest valence band at the $K$ point [13,24,47]. In this range of energy, the partial density of states shows a maximum that could explain the resonance behavior observed in Raman scattering. This hypothesis needs to be further clarified on a theoretical point of view and/or with Raman scattering experiments performed in the UV range of energy. Finally, in contrast to the case of optical phonon modes described above, the LA phonon replicas show a pronounced resonance only with the low-energy $A$ and $B$ modes, and are only weakly visible when exciting at higher energy. This behavior is shown in Fig. 4(e) for the 2L- and 4L-MoSe 2 . Similar to the case of $\mathrm{MoS}_{2}$ [15], when using an excitation energy close to that of $A$ or $B$ excitons, these acoustical phonon replicas can become the dominant contribution to Raman scattering spectrum of $\mathrm{MoSe}_{2}$ (see spectrum at $676 \mathrm{~nm}$ in Fig. 1 and Appendix).

The low-energy resonance of the $A_{1}^{\prime} / A_{1 g}$ mode is presented in more detail in Fig. 5(a) for excitation energies between 1.4 and $1.8 \mathrm{eV}$. The photoluminescence (PL) of $\mathrm{MoSe}_{2}$ monolayers arises from the low-energy $A$ exciton [48]. In Fig. 5(b), the room-temperature PL spectra recorded on the same samples are plotted for comparison with the data displayed in Fig. 5(a).

When increasing the number of layers, we observe at room temperature a marked decrease of the luminescence intensity together with a small shift of the luminescence energy, from $1.57 \mathrm{eV}$ for $1 \mathrm{~L}-\mathrm{MoSe}_{2}$, to $1.54 \mathrm{eV}$ for $2 \mathrm{~L}-\mathrm{MoSe}_{2}$, and to $1.52 \mathrm{eV}$ for $3 \mathrm{~L}-$ and $4 \mathrm{~L}-\mathrm{MoSe}_{2}$. The origin of these two effects is still debated. They could originate (i) from a directto-indirect band-gap transition when going from monolayers to multilayers, or (ii) reflect the difference in the relative

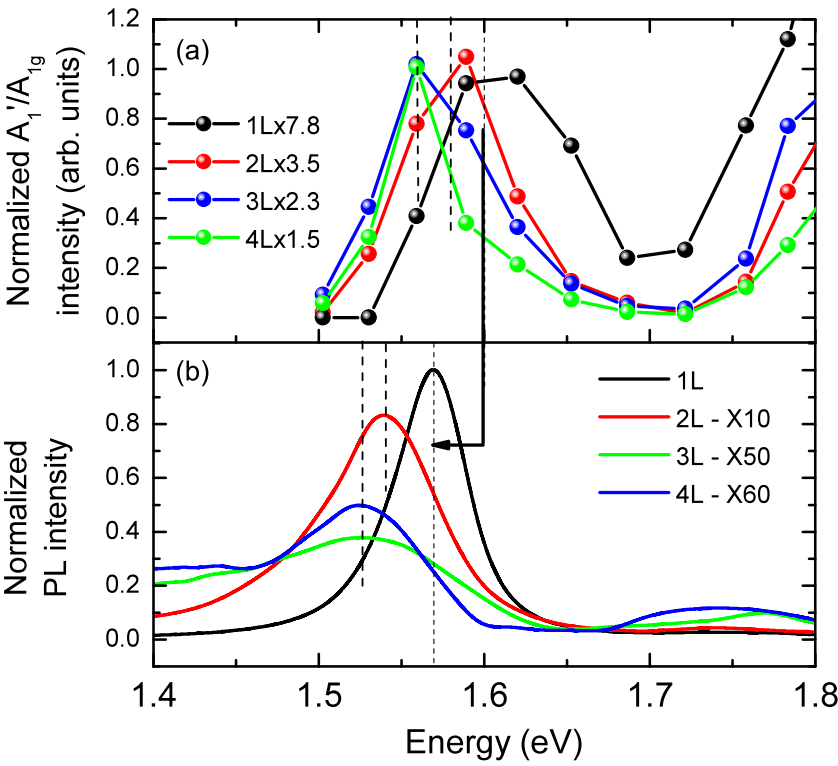

FIG. 5. (a) Normalized intensity of the $A_{1}$ mode at $242 \mathrm{~cm}^{-1}$ as a function of the excitation laser energy. (b) Photoluminescence intensity, normalized to the maximum intensity of the monolayer. Vertical dashed lines indicate the observed maxima of Raman intensities (a) and of luminescence (b). Note the shift between the two maxima (highlighted with the vertical arrow, and equal to the energy of the involved phonon), indicative of an outgoing resonance process.

energies of the $A$ exciton and of the single-particle indirect band gap due to the change of dielectric screening and the decrease of the exciton binding energy for multilayers. The observed resonances for the $A_{1}^{\prime} / A_{1 g}$ Raman scattering feature are centered at $1.610 \pm 0.01,1.575 \pm 0.003,1.560 \pm 0.002$, and $1.560 \pm 0.002 \mathrm{eV}$ for $1 \mathrm{~L}$ - to $4 \mathrm{~L}-\mathrm{MoSe}_{2}$, respectively. We interpret the energy difference between the emission of $A$ excitons and the resonance in the Raman scattering as a signature of an efficient outgoing resonance. In fact, the maximum intensity of the Raman scattering is observed in this material when the incoming photon energy is equal to the sum of the exciton and of the scattered phonon energies.

As already mentioned, layered materials also show lowenergy shear phonon modes, of the $E$-type symmetry, and breathing phonon modes, of the $A$-type symmetry. Detailed investigation of these modes in few-layer samples has recently become more accessible due to the commercial development of volume Bragg grating filters allowing to easily explore the low-energy range [49] of the Raman scattering spectrum, down to $5-10 \mathrm{~cm}^{-1}$. The shear and breathing modes are today the subject of intense research in the field of $M X_{2}$. Their Raman shift as a function of the number of layers and their polarization selection rules have been described in detail for $\mathrm{MoS}_{2}, \mathrm{WSe}{ }_{2}, \mathrm{MoSe}_{2}, \mathrm{WS}_{2}$, and in $\mathrm{MoTe}_{2}$ [29,34,35,44]. Because of technical difficulties, such studies are usually performed at a single excitation wavelength, and a complete picture of the excitation profile of such low-energy modes in $M X_{2}$ is still lacking. Resonant Raman spectroscopy of such low-energy modes has been performed in semimetallic twisted graphene, allowing to trace the evolution of its high-energy 


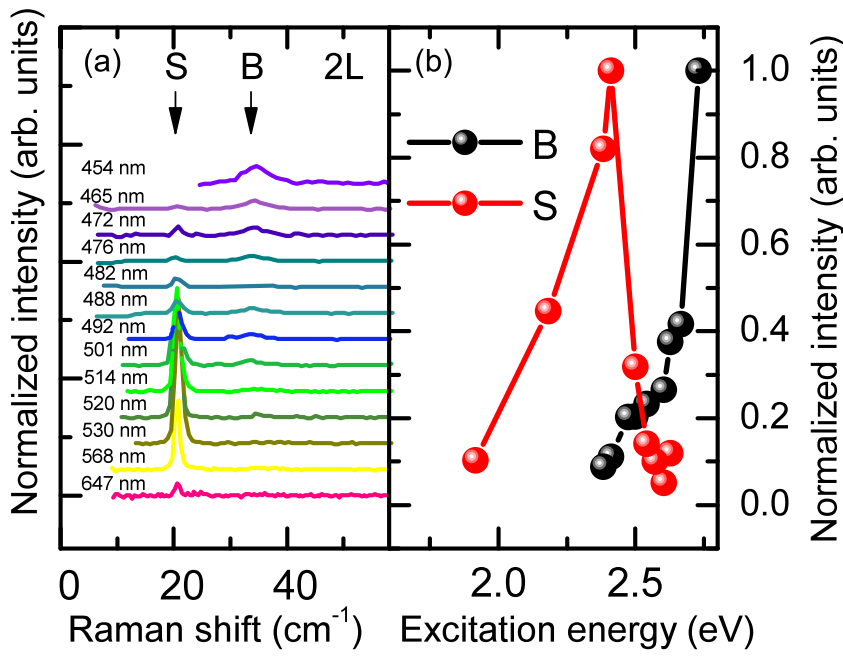

FIG. 6. (a) Raman scattering spectra of $2 \mathrm{~L}-\mathrm{MoSe}_{2}$ for excitations energy ranging from 458 to $647 \mathrm{~nm}$ showing the shear (S) and breathing (B) modes. (b) Evolution of the normalized intensity of the shear mode (red dots) and of the breathing mode (black dots) as a function of the excitation laser energy.

bands and to quantify the interlayer interaction as a function of the twist angle [50].

The use of a triple spectrometer and the access to large number of laser lines has allowed us to perform such a detailed resonant study for monolayer and multilayer $\mathrm{MoSe}_{2}$. In Fig. 6(a), we present Raman scattering spectra of the shear (S) and breathing (B) modes of $2 \mathrm{~L}-\mathrm{MoSe}_{2}$ measured for excitation wavelengths from 454 to $647 \mathrm{~nm}$. They are observed at 21 and $34 \mathrm{~cm}^{-1}$, respectively [44], and their energies do not change with the excitation wavelength. Figure 6(b) illustrates the evolution of the normalized intensity of these two modes as a function of the excitation laser energy. As can be seen, they show rather distinct resonance behaviors. The $E$-type symmetry shear mode exhibits a pronounced resonance close to $\sim 2.4 \mathrm{eV}$, similar to the resonance observed for both high-energy $A$-type modes, which correspond to the $C$-exciton energy in $2 \mathrm{~L}-\mathrm{MoSe}_{2}$. In contrast, the breathing mode, of the $A$-type symmetry, does not show this resonance but its intensity increases with increasing excitation laser energy. At excitation energies below $2.4 \mathrm{eV}$, the intensity of the breathing mode is nearly not observable in $2 \mathrm{~L}-\mathrm{MoSe}_{2}$, grows rapidly at higher energies, becoming stronger than that of the shear mode, and eventually representing the most pronounced low-energy feature above $2.65 \mathrm{eV}$. For excitation energies lower than $1.95 \mathrm{eV}(647 \mathrm{~nm})$, we could not observe these low-energy modes mainly because of the strong low-energy scattering and/or PL when approaching the energies of the $A$ and $B$ excitons. Similar to the TO $E^{\prime} / E_{g}$ at $289 \mathrm{~cm}^{-1}$ and to the $A_{2}^{\prime \prime} / A_{1 g}$ mode at $354 \mathrm{~cm}^{-1}$, the breathing mode's intensity seems to show a resonance with electronic excitations above the energy of the $C$ exciton. For different $M X_{2}$ materials, depending on the excitation laser and on the exciton energy structure, very different intensity ratios between the shear and breathing modes have been reported recently $[19,29,34,35,44]$. As follows from the study reported here, these differences arise from the peculiar resonance effect affecting differently the two modes.

To conclude, we have presented a comprehensive Raman scattering study of $\mathrm{MoSe}_{2}$ monolayers and multilayers. We have shown in this compound the existence of new Raman modes which are only active in multilayers, and described their resonances when tuning the excitation laser energy across the three different excitons of this material. Resonant Raman spectroscopy appears as a well-adapted tool to study a variety of excitons in thin layers of $M X_{2}$ and their coupling to optical phonons. Our experiments confirm the lowering of the $A$ exciton's energy when increasing the number of layers and this result is corroborated by the PL measurements. We also show symmetry-dependent exciton-phonon coupling, in particular, we show experimentally that the low-energy shear mode of a bilayer displays a a pronounced resonance with the $C$ exciton while the breathing mode does not. On the other hand, this latter mode, together with the $E^{\prime} / E_{g}$ and $A_{2}^{\prime \prime} / A_{1 g}$ modes, have an intensity that grows with the excitation laser energy, which is a signature of a still unexplored resonance at higher energy, in the deep UV range.

We acknowledge fruitful discussions with $\mathrm{S}$. Berciaud, M. Molas, and with M. Potemski. Part of this work has been supported by the TWINFUSYON project, by the ERC Advanced Grant MOMB (Contract No. 320590) and by the EC Graphene Flagship (Project No. 604391). Samples were fabricated at the Nanofab facility at Institut Néel-CNRS.

\section{APPENDIX}

\section{Photograph of the different $\mathrm{MoSe}_{2}$ specimens}

Large flakes of $\mathrm{MoSe}_{2}$ monolayers and multilayers have been produced by mechanical exfoliation of bulk $\mathrm{MoSe}_{2}$. Flakes were then deposited on a $\mathrm{Si} / \mathrm{SiO}_{2}$ substrate with $90 \mathrm{~nm}$ of $\mathrm{SiO}_{2}$. The substrate had been patterned with circular holes of $6 \mu \mathrm{m}$ diameter, but all the results presented in the main text have been obtained on supported regions. The following photograph in Fig. 7 presents the different specimens that were used for the reported measurements.

\section{Raman scattering features at 242 and $252 \mathrm{~cm}^{-1}$}

As stated in the main text, $\mathrm{MoSe}_{2}$ monolayers can be identified by (i) a single component $A_{1}^{\prime}$ feature at $240 \mathrm{~cm}^{-1}$ while this feature is observed at $242.5 \mathrm{~cm}^{-1}$ for $2 \mathrm{~L}$ and is a multicomponent feature for $N>2$, (ii) the nonobservation of the two $E^{\prime \prime} / E_{1 g}$ and $A_{2}^{\prime \prime} / A_{1 g}$ modes at 170 and $354 \mathrm{~cm}^{-1}$, respectively, and (iii) by the observation of an energy separation bigger than $9 \mathrm{~cm}^{-1}$ between the $A_{1}^{\prime}$ and the feature close to $252 \mathrm{~cm}^{-1}$ (see Fig. 8). These are the three Raman scattering signatures of $1 \mathrm{~L}-\mathrm{MoSe}_{2}$ that emerge from this study.

\section{Spectra normalization procedure}

The normalization of Raman scattering intensities is crucial for the study of resonance effects. All intensities presented in the main text are normalized according to the following procedure: We first make a background correction. This background is most of the case flat but, when the excitation wavelength 

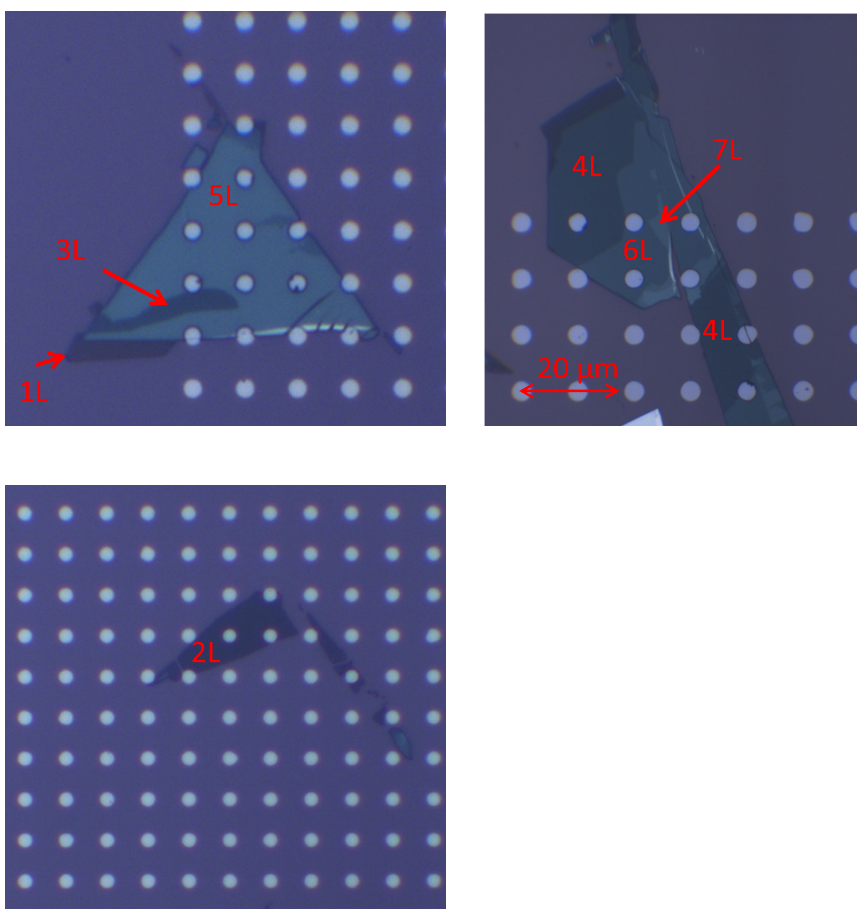

FIG. 7. Three optical photographs of the same wafer with different locations corresponding to $1 \mathrm{~L}-, 2 \mathrm{~L}-, 3 \mathrm{~L}-, 4 \mathrm{~L}-, 5 \mathrm{~L}-$, and $6 \mathrm{~L}-\mathrm{MoSe}_{2}$ specimens.

is set close to the low-energy excitons of monolayer or few-layer $\mathrm{MoSe}_{2}$, luminescence signals affect the spectra and are removed by using standard computer tools. Spectra are then normalized by the integrated silicon signal at $522 \mathrm{~cm}^{-1}$. The silicon first-order Raman scattering peak shows a resonance when using high excitation energies. This effect was studied in detail in Ref. [32] and has to be taken into account by normalizing the measured signal by the resonance curve of silicon (which also includes the wavelength dependence of the Raman scattering process). Finally, the flake is on top of a $\mathrm{SiO}_{2} / \mathrm{Si}$ structure which produces optical interferences which have a strong impact on the measured Raman intensities. As it is shown in the literature [17,33,51], the Raman intensities of the $\mathrm{Si}$ and the $\mathrm{MoSe}_{2}$ layers are proportional to an enhancement factor due to the optical interference existent in a multilayered structure given by

$$
I_{\mathrm{MoSe}_{2}}=\int_{0}^{d}\left|E_{e x}(x) E_{s c}(x)\right|^{2} d x,
$$

where $E_{e x}(x)$ and $E_{s c}(x)$ are the electrical field's amplitude of the total incident and scattered light, respectively, and $d$ is the thickness of the $\mathrm{MoSe}_{2}$. The enhancement factor for the $\mathrm{Si}$ substrate is $[17,33]$

$$
I_{\mathrm{Si}}=\int_{0}^{\infty}\left|F_{e x}(x) F_{s c}(x)\right|^{2} d x,
$$

where $F_{e x}(x)$ and $F_{s c}(x)$ are the electrical field amplitude of the total incident and scattered light, respectively. It is important to take into account the wavelength dependence of the complex refractive index of each material to describe the propagation of the incident and of the scattered light. In the experiment, the incident wavelength is given by the exciting
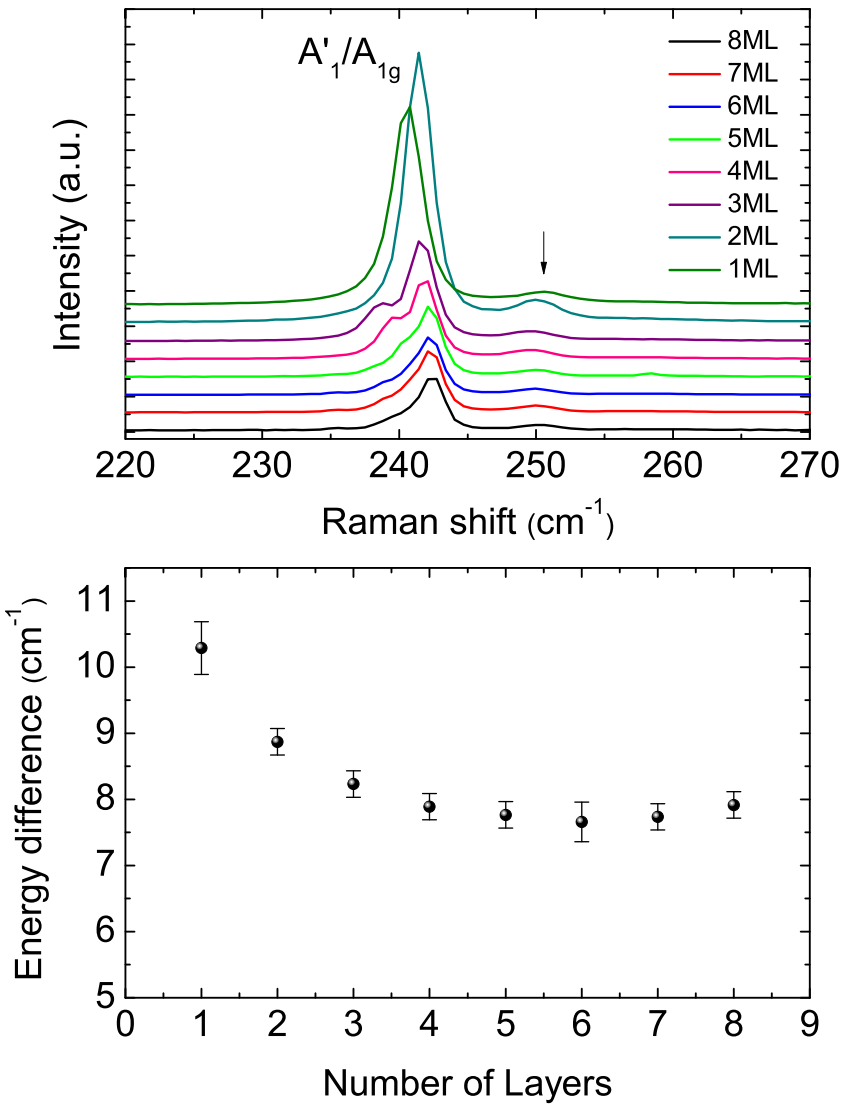

FIG. 8. Upper panel: Raman scattering spectra for $1 \mathrm{~L}-$ to $8 \mathrm{~L}-\mathrm{MoSe}_{2}$ samples. Lower panel: energy difference between the $252-\mathrm{cm}^{-1}$ feature and the $A_{1}^{\prime} / A_{1 g}$ feature. The energy separation between these modes is maximum in the case of the monolayer and then decreases for thicker samples. The arrow in the top panel indicates the Raman scattering feature observed close to $250 \mathrm{~cm}^{-1}$.

laser and the Raman process defines the scattered wavelength. To correct the data, we multiply the experimental spectra by the ratio $I_{\mathrm{Si}} / I_{\mathrm{MoSe}_{2}}[17,33]$ after performing the silicon signal normalization described above.

Expression (A2) is calculated considering a "unidimensional" problem, where the Si substrate is treated as a halfinfinite media. In the IR region, where the $\mathrm{Si}$ absorption is small, when using a microscope objective with a large numerical aperture, we should take the divergence effect of the focused Gaussian beam into account and calculate the integral in a probed volume defined by the depth of focus. The depth of focus $\Delta f$ is defined as the distance between the points at each side of the beam waist where the irradiance of the beam falls to one-half of its value [52]. According to Ref. [52], it is given by

$$
\Delta f=\frac{\pi\left(2 w_{0}\right)^{2}}{2 \lambda},
$$

where $w_{0}$ is the beam radius at the waist (spot size in the focus) and $\lambda$ is the wavelength within the material. Taking that into account, the upper integral $\operatorname{limit}(\infty)$ must be modified to $\Delta f / 2$. 

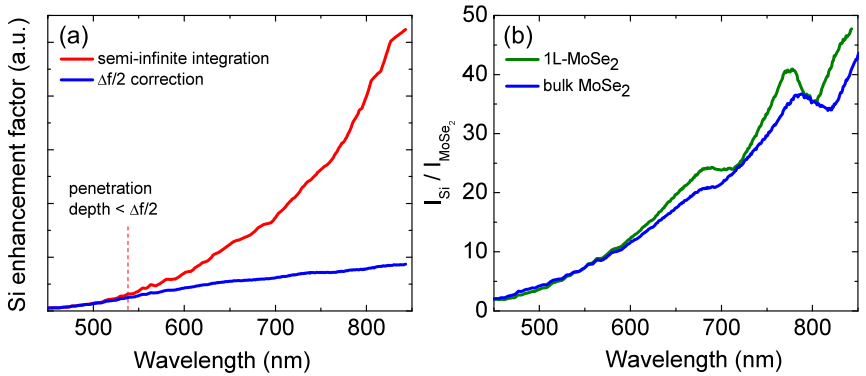

FIG. 9. (a) Interference enhancement factor for the Si Raman signal $\left(I_{\mathrm{Si}}\right)$ as a function of wavelength for a semi-infinite integration and the $\Delta f / 2$ correction. When the penetration depth is smaller than $\Delta f / 2(400-550 \mathrm{~nm})$, the $I_{\mathrm{Si}}$ factor is approximately the same for both calculations. (b) Ratio $I_{\mathrm{Si}} / I_{\mathrm{MoSe}_{2}}$ for $1 \mathrm{~L}-\mathrm{MoSe}_{2}$ and for bulk $\mathrm{MoSe}_{2}$.
For $1 \mathrm{~L}-\mathrm{MoSe}_{2}$ sample, Fig. 9(a) shows the comparison between the semi-infinite integration and the $\Delta f / 2$ correction for the Si Raman intensity both as a function of wavelength. Here, we assumed a Raman shift of $520 \mathrm{~cm}^{-1}$. The complex refractive index for $\mathrm{Si}_{\text {and }} \mathrm{SiO}_{2}$ as a function of the wavelength was taken from Refs. [53,54], respectively, and the refractive index of $1 \mathrm{~L}-\mathrm{MoSe}_{2}$ was taken from Ref. [26]. Figure 9(b) shows the ratio of the $\mathrm{Si}$ and the $\mathrm{MoSe}_{2}$ enhancement factor as a function of the wavelength for a $1 \mathrm{~L}-\mathrm{MoSe}_{2}$ and bulk $\mathrm{MoSe}_{2}$ assuming a Raman shift of $290 \mathrm{~cm}^{-1}$. The bulk $\mathrm{MoSe}_{2}$ refractive index was taken from Ref. [33]. As can be seen, the enhancement factor for $1 \mathrm{~L}-\mathrm{MoSe}_{2}$ and bulk $\mathrm{MoSe}_{2}$ are similar so that we used the refractive index of the $1 \mathrm{~L}-\mathrm{MoSe}_{2}$ for the calculations in the multilayer samples.
[1] K. F. Mak, C. Lee, J. Hone, J. Shan, and T. F. Heinz, Phys. Rev. Lett. 105, 136805 (2010).

[2] Q. Wang, K. Kalantar-Zadeh, A. Kis, J. Coleman, and M. Strano, Nat. Nanotechnol. 7, 699 (2012).

[3] D. Xiao, G.-B. Liu, W. Feng, X. Xu, and W. Yao, Phys. Rev. Lett. 108, 196802 (2012).

[4] X. Xu, W. Yao, D. Xiao, and T. F. Heinz, Nat. Phys. 10, 343 (2014).

[5] B. Radisavljevic, A. Radenovic, J. Brivio, V. Giacometti, and A. Kis, Nat. Nanotechnol. 6, 147 (2011).

[6] Z. Yin, H. Li, H. Li, L. Jiang, Y. Shi, Y. Sun, G. Lu, Q. Zhang, X. Chen, and H. Zhang, ACS Nano 6, 74 (2012).

[7] T. Georgiou, R. Jalil, B. D. Belle, L. Britnell, R. V. Gorbachev, S. V. Morozov, Y.-J. Kim, A. Gholinia, S. J. Haigh, O. Makarovsky, L. Eaves, L. A. Ponomarenko, A. K. Geim, K. S. Novoselov, and A. Mishchenko, Nat. Nanotechnol. 8, 100 (2013).

[8] A. K. Geim and I. V. Grigorieva, Nature (London) 499, 419 (2013).

[9] F. Withers, O. Del Pozo-Zamudio, A. Mishchenko, A. P. Rooney, A. Gholinia, K. Watanabe, T. Taniguchi, S. J. Haigh, A. K. Geim, A. I. Tartakovskii, and K. S. Novoselov, Nat. Mater. 14, 301 (2015).

[10] K. He, N. Kumar, L. Zhao, Z. Wang, K. F. Mak, H. Zhao, and J. Shan, Phys. Rev. Lett. 113, 026803 (2014).

[11] A. Chernikov, T. C. Berkelbach, H. M. Hill, A. Rigosi, Y. Li, O. B. Aslan, D. R. Reichman, M. S. Hybertsen, and T. F. Heinz, Phys. Rev. Lett. 113, 076802 (2014).

[12] G. Scholes and G. Rumbles, Nat. Mater. 5, 683 (2006).

[13] M. M. Ugeda, A. J. Bradley, S.-F. Shi, F. H. da Jornada, Y. Zhang, D. Y. Qiu, W. Ruan, S.-K. Mo, Z. Hussain, Z.-X. Shen, F. Wang, S. G. Louie, and M. F. Crommie, Nat. Mater. 13, 1091 (2014).

[14] C. Lee, H. Yan, L. E. Brus, T. F. Heinz, J. Hone, and S. Ryu, ACS Nano 4, 2695 (2010).

[15] K. Gołasa, M. Grzeszczyk, P. Leszczyński, C. Faugeras, A. A. L. Nicolet, A. Wysmołek, M. Potemski, and A. Babiński, Appl. Phys. Lett. 104, 092106 (2014).

[16] E. del Corro, H. Terrones, A. Elias, C. Fantini, S. Feng, M. A. Nguyen, T. E. Mallouk, M. Terrones, and M. A. Pimenta, ACS Nano 8, 9629 (2014).
[17] B. R. Carvalho, L. M. Malard, J. M. Alves, C. Fantini, and M. A. Pimenta, Phys. Rev. Lett. 114, 136403 (2015).

[18] N. Scheuschner, R. Gillen, M. Staiger, and J. Maultzsch, Phys. Rev. B 91, 235409 (2015).

[19] J.-U. Lee, J. Park, Y.-W. Son, and H. Cheong, Nanoscale 7, 3229 (2015).

[20] T. Sekine, M. Izumi, T. Nakashizu, K. Uchinokura, and E. Matsuura, J. Phys. Soc. Jpn. 49, 1069 (1980).

[21] S. Tongay, J. Zhou, C. Ataca, K. Lo, T. S. Matthews, J. Li, J. C. Grossman, and J. Wu, Nano Lett. 12, 5576 (2012).

[22] P. Tonndorf, R. Schmidt, P. Böttger, X. Zhang, J. Börner, A Liebig, M. Albrecht, C. Kloc, O. Gordan, D. R. T. Zahn, S. M. de Vasconcellos, and R. Bratschitsch, Opt. Express 21, 4908 (2013).

[23] N. Kumar, Q. Cui, F. Ceballos, D. He, Y. Wang, and H. Zhao, Nanoscale 6, 4915 (2014).

[24] S. Horzum, H. Sahin, S. Cahangirov, P. Cudazzo, A. Rubio, T. Serin, and F. M. Peeters, Phys. Rev. B 87, 125415 (2013).

[25] A. Molina-Sánchez and L. Wirtz, Phys. Rev. B 84, 155413 (2011).

[26] Y. Li, A. Chernikov, X. Zhang, A. Rigosi, H. M. Hill, A. M. van der Zande, D. A. Chenet, E.-M. Shih, J. Hone, and T. F. Heinz, Phys. Rev. B 90, 205422 (2014).

[27] M. Yamamoto, S. T. Wang, M. Ni, Y.-F. Lin, S.-L. Li, S. Aikawa, W.-B. Jian, K. Ueno, K. Wakabayashi, and K. Tsukagoshi, ACS Nano 8, 3895 (2014).

[28] C. Ruppert, O. B. Aslan, and T. F. Heinz, Nano Lett. 14, 6231 (2014).

[29] G. Froehlicher, E. Lorchat, F. Fernique, C. Joshi, A. MolinaSanchez, L. Wirtz, and S. Berciaud, Nano Lett. (to be published).

[30] X. Luo, Y. Zhao, J. Zhang, M. Toh, C. Kloc, Q. Xiong, and S. Y. Quek, Phys. Rev. B 88, 195313 (2013).

[31] H. Terrones, E. Del Corro, S. Feng, J. M. Poumirol, D. Rhodes, D. Smirnov, N. R. Pradhan, Z. Lin, M. A. T. Nguyen, A. L. Elías, T. E. Mallouk, L. Balicas, M. A. Pimenta, and M. Terrones, Sci. Rep. 4, 4215 (2014).

[32] J. B. Renucci, R. N. Tyte, and M. Cardona, Phys. Rev. B 11, 3885 (1975). 
[33] S.-L. Li, H. Miyazaki, H. Song, H. Kuramochi, S. Nakaharai, and K. Tsukagoshi, ACS Nano 6, 7381 (2012).

[34] X. Zhang, W. P. Han, J. B. Wu, S. Milana, Y. Lu, Q. Q. Li, A. C. Ferrari, and P. H. Tan, Phys. Rev. B 87, 115413 (2013).

[35] Y. Zhao, X. Luo, H. Li, J. Zhang, P. T. Araujo, C. K. Gan, J. Wu, H. Zhang, S. Y. Quek, M. S. Dresselhaus, and Q. Xiong, Nano Lett. 13, 1007 (2013).

[36] D. J. Late, S. N. Shirodkar, U. V. Waghmare, V. P. Dravid, and C. N. R. Rao, ChemPhysChem 15, 1592 (2014).

[37] A. Berkdemir, H. R. Gutíerrez, A. R. Botello-Méndez, N. PereaLópez, A. L. Elías, C.-I. Chia, B. Wang, V. H. Crespi, F. LópezUrías, J.-C. Charlier, H. Terrones, and M. Terrones, Sci. Rep. 3, 1755 (2013).

[38] B. Chakraborty, H. Matte, A. Sood, and C. Rao, J. Raman Spectrosc. 44, 92 (2013).

[39] K. Gołasa, M. Grzeszczyk, R. Bożek, P. Leszczyński, A. Wysmołek, M. Potemski, and A. Babiński, Solid State Commun. 197, 53 (2014).

[40] J.-U. Lee, K. Kim, and H. Cheong, 2D Mater. 2, 044003 (2015).

[41] D. Y. Qiu, F. H. da Jornada, and S. G. Louie, Phys. Rev. Lett. 111, 216805 (2013).

[42] A. J. Bradley, M. M. Ugeda, F. H. da Jornada, D. Y. Qiu, W. Ruan, Y. Zhang, S. Wickenburg, A. Riss, J. Lu, S.-K. Mo, Z. Hussain, Z.-X. Shen, S. G. Louie, and M. F. Crommie, Nano Lett. 15, 2594 (2015).

[43] J. L. Verble and T. J. Wieting, Phys. Rev. Lett. 25, 362 (1970).
[44] S.-Y. Chen, C. Zheng, M. S. Fuhrer, and J. Yan, Nano Lett. 15, 2526 (2015).

[45] A. Arora, M. Koperski, K. Nogajewski, J. Marcus, C. Faugeras, and M. Potemski, Nanoscale 7, 10421 (2015).

[46] A. Arora, K. Nogajewski, M. Molas, M. Koperski, and M. Potemski, Nanoscale 7, 20769 (2015).

[47] G. Wang, C. Robert, A. Suslu, B. Chen, S. Yang, S. Alamdari, I. C. Gerber, T. Amand, X. Marie, S. Tongay, and B. Urbaszek, Nat. Commun. 6, 10110 (2015).

[48] D. MacNeill, C. Heikes, K. F. Mak, Z. Anderson, A. Kormányos, V. Zólyomi, J. Park, and D. C. Ralph, Phys. Rev. Lett. 114, 037401 (2015).

[49] P. H. Tan, W. P. Han, W. J. Zhao, Z. H. Wu, K. Chang, H. Wang, Y. F. Wang, N. Bonini, N. Marzari, N. Pugno, G. Savini, A. Lombardo, and A. C. Ferrari, Nat. Mater. 11, 294 (2012).

[50] J.-B. Wu, X. Zhang, M. Ijaes, W.-P. Han, X.-F. Qiao, X.-L. Li, D.-S. Jiang, A. C. Ferrari, and P.-H. Tan, Nat. Commun. 5, 5309 (2014).

[51] D. Yoon, H. Moon, Y.-W. Son, J. S. Choi, B. H. Park, Y. H. Cha, Y. D. Kim, and H. Cheong, Phys. Rev. B 80, 125422 (2009).

[52] J. A. M. B. M. Delhaye, J. Barbillat, and E. D. Silva, Raman Microscopy, Development and Applications (Academic, San Diego, 1996).

[53] E. Palik, Handbook of Optical Constants of Solids, Vol. 3 (Academic, San Diego, 1998).

[54] I. H. Malitson, J. Opt. Soc. Am. 55, 1205 (1965). 